\title{
Novel Mystery of Double Slit/Cross-double Slit Experiments-Curved Interference Pattern andlnterference Pattern Depending on Orientation of Diaphragm
}

hui peng ( $\sim$ davidpeng1749@gmail.com )

\section{Research Article}

Keywords: double slit experiments, cross-double slit experiments, interference pattern, wave interpretation, quantum mechanics, optics

Posted Date: July 9th, 2021

DOl: https://doi.org/10.21203/rs.3.rs-701216/v1

License: (c) (i) This work is licensed under a Creative Commons Attribution 4.0 International License.

Read Full License 


\title{
Novel Mystery of Double Slit/Cross-double Slit Experiments --- Curved Interference Pattern and Interference Pattern Depending on Orientation of Diaphragm
}

\author{
Hui Peng \\ Email: davidpeng1749@gmail.com
}

\begin{abstract}
Young's double slit experiments express the mystery of quantum mechanics. To explore the mystery, varieties of the double slit and cross-double slit experiments were performed. In this article, we show novel mystery phenomena: (1) the characteristics of the interference patterns of the double slit and cross-double slit experiments depend on the orientation of the diaphragm used in the experiment, specifically on which axis the diaphragm rotates around, the rotating angle and how to rotate, clockwise or counterclockwise; and (2) in the cross-double slit experiments, the characteristics of the interference patterns also depend on which slit photons pass through; (3) unlike the regular double slit and regular cross-double slit experiments, in which the interference patterns are along the straight line, the interference patterns of the double slit/cross-double slit experiments can be along curve, and the curvatures depend on the orientations of the diaphragms of the double slit/cross-double slit. Those experiments show more mysteries of the double slit and cross-double slit experiments and provide comprehensive data for developing/testing a theoretical model.
\end{abstract}

Keywords: double slit experiments, cross-double slit experiments, interference pattern, wave interpretation

Declaration: The author declares that there is no potential conflict of interest

\section{Content}

1. Introduction

2. Apparatus

3. Novel Characteristics of Interference Patterns Depending on Orientation of Diaphragm

3.1. Rotating Diaphragm Around Y-Axis: Curved Interference patterns

3.1.1. Rotating Diaphragm of Double Slit

3.1.2. Rotating Diaphragm of Double Slit Continuously (Appendix-1 Video)

3.1.3. Rotating Diaphragm of Cross-Double Slit

(A) Three Double Slits Crossing; (B) Two Double Slits Crossing

3.1.4. Rotating Diaphragm of Triple Slit 


\subsubsection{Rotating Diaphragm of Single Slit}

\subsection{Rotating Diaphragm Around Z-Axis: Orientation-dependence of Interference Pattern}

3.2.1. Derivation of Orientation-dependence Formular of Interference Pattern of Double Slit

3.2.2. Rotating Diaphragm of Double Slit

3.2.3. Rotating Diaphragm of Cross-Double Slit

(A) Two Double Slits Crossing; (B) Six Double Slits Crossing;

(C) Three Double Slits Crossing

3.2.4. Rotating Diaphragm of Triple Slit

3.3. Rotating Diaphragm Around X-Axis (Normal Vector): Rotation-Invariance

3.3.1. Rotating Diaphragm of Double Slit

3.3.2. Rotating Diaphragm of Cross-Double Slit

4. Summary

Appendix

A-1 Video: Evolution of Curved Interference Pattern of Double Slit Experiment

A-2 Novel Multi-Slits for Exploring Mystery of Cross-Double Slit

\section{Introduction}

Young's double slit experiment was first performed in 1801 [1] [2], which, 100 years later, led to wave-particle duality. Feynman called it "a phenomenon which is impossible $[\ldots]$ to explain in any classical way, and which has in it the heart of quantum mechanics. In reality, it contains the only mystery [of quantum mechanics]." [3]. Moreover, the nature of photons truly puzzled Einstein. He wrote to M. Besso: "All these 50 years of conscious brooding have brought me no nearer to the answer to the question: What are light quanta?" [4].

In the double slit experiments, the characteristics of the interference pattern is determined by three factors, wave length, the spacing between two slits and distance between the double slit and screen, as described by equation, $y_{\text {const }}=m \frac{\lambda}{d} L$.

Note that (1) there is only one factor related to the parameter of the diaphragm of the double slit, the spacing $d$; (2) the fringes of the interference pattern distribute along a straight line.

We raise a question that whether there are other factors related with the diaphragm affect the characteristics of the interference pattern? To answer this question, we emphasize that in the standard double slit experiments, the light beam is perpendicular to the plane of the diaphragm of the double slit.

We are interested in how the interference patterns vary with different orientations of the diaphragm of double slit/cross-double slit. We study the orientation-dependence of the interference pattern of a 
standard double-slit/cross-double slit rotating around the $\mathrm{x}$-axis, $\mathrm{y}$-axis, and z-axis, respectively.

Recently, to further explore the mystery, it has been shown that the photons' behaviors/interference pattern depend on the orientations of the diaphragms of the double slit and cross-double slit [5] [6].

We show in detail the dependance of the interference patterns on the orientations of diaphragms.

\section{Apparatus}

The experiments utilize a laser source, the diaphragms of the double slit (Figure 1a) and cross-double slit (Figure 1b), a protractor (Figure 1c) and a screen/detector.

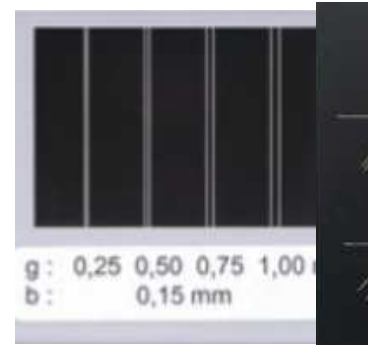

(a)

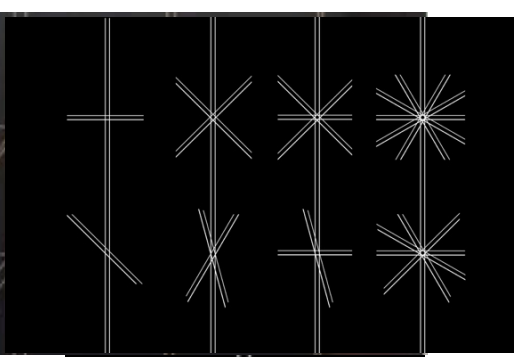

(b)

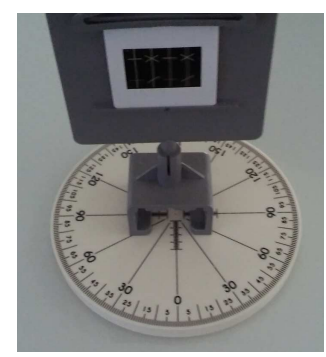

(c)

Figure 1 Apparatus

We study the orientation-dependence of the interference pattern of the double slit and cross-double slit rotating, either clockwise or counterclockwise, different angles around the X, Y and Z-axis.

\section{Novel Characteristics of Interference Patterns Depending on Orientation of Diaphragm}

\subsection{Rotating Diaphragm Around Y-Axis: Curved Interference patterns}

\subsubsection{Rotating Diaphragm of Double Slit Around Y-Axis}

The schematic of the experimental setup for the double slit experiments is shown in Figure 2.

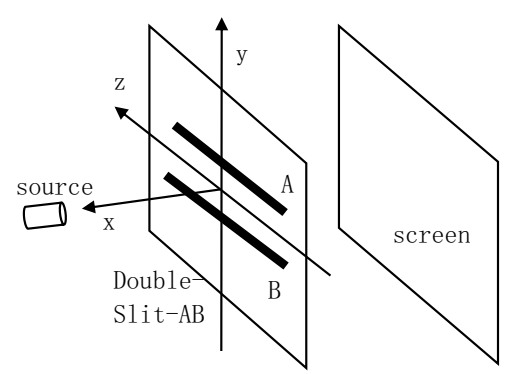

Figure 2 Schematic drawing of apparatus

The double-slit-AB is in the $\mathrm{y}-\mathrm{z}$ plane, slit $\mathrm{A}$ and slit $\mathrm{B}$ are along the $\mathrm{z}$-axis, its normal vector is along the $\mathrm{x}$-axis and points to source, the spacing between slits A and B is " $\mathrm{d}$ ", and photons travel along the negative $\mathrm{x}$ direction. Its interference pattern is shown in Figure 3a.

The standard optical equation, $y_{\text {const }}=m \frac{\lambda}{d} L$, describe the interference pattern of the double slit experiments. This equation is derived for a special situation that the source is on the normal vector of the plane of the double slit. We study how the characteristics of interference patterns vary with the different orientations of the diaphragm of the double slit. 
Experiment-1: rotating the double slit-AB clockwise around Y-axis with different discrete angles, $45^{\circ}$, $60^{\circ}$ and $75^{\circ}$. The original orientation corresponds to $0^{0}$ rotation.
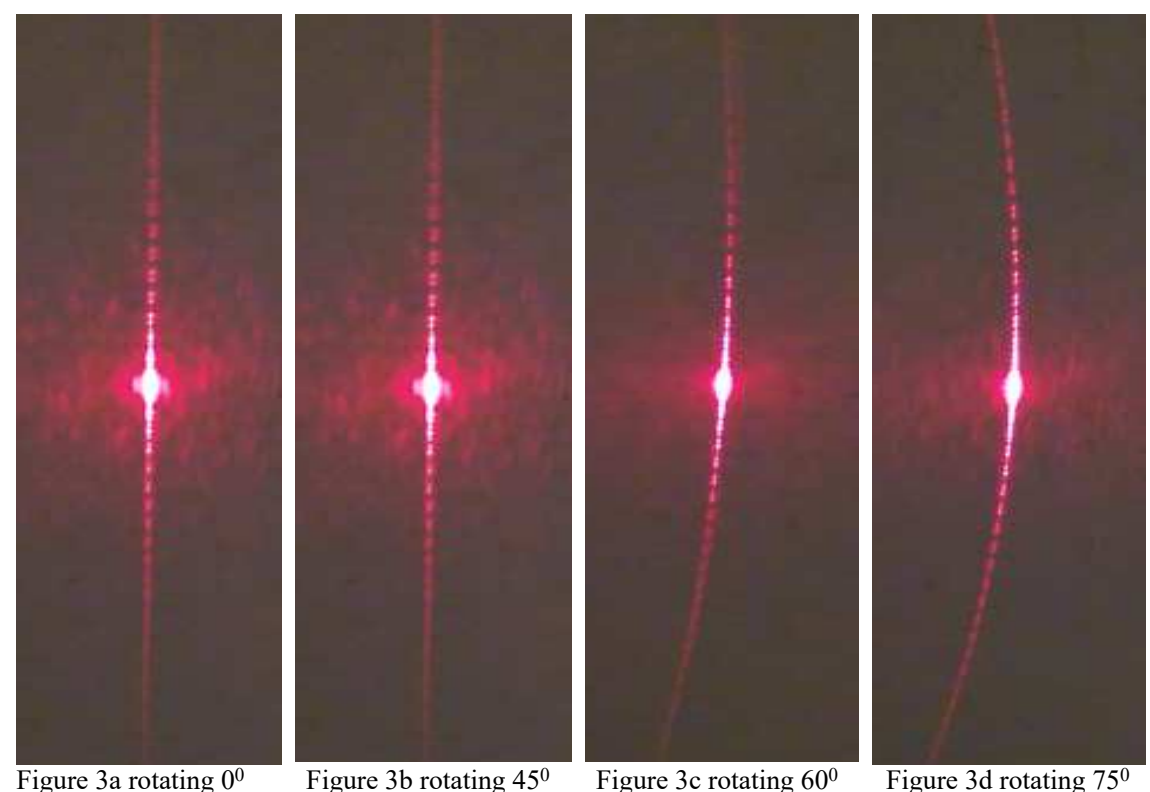

Observation (Figure 3): the interference patterns curved towards the left side. The larger the rotation angle, the smaller curvature of the interference patterns.

Experiment-2: rotating the double slit counterclockwise with different discrete angles (Figure 4):

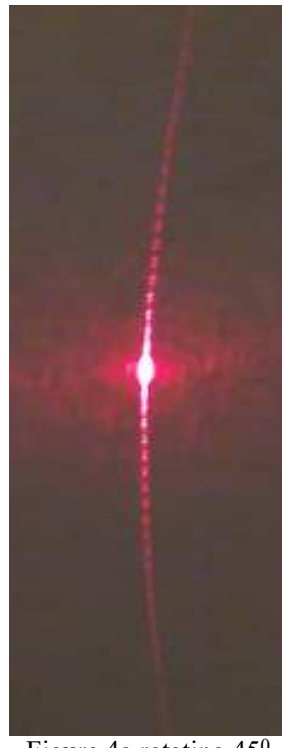

Figure 4 a rotating $45^{0}$

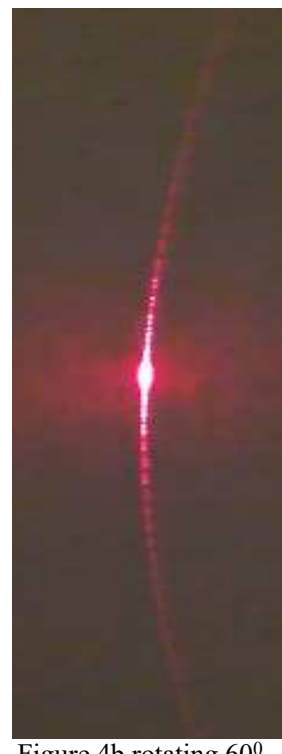

Figure $4 \mathrm{~b}$ rotating $60^{0}$

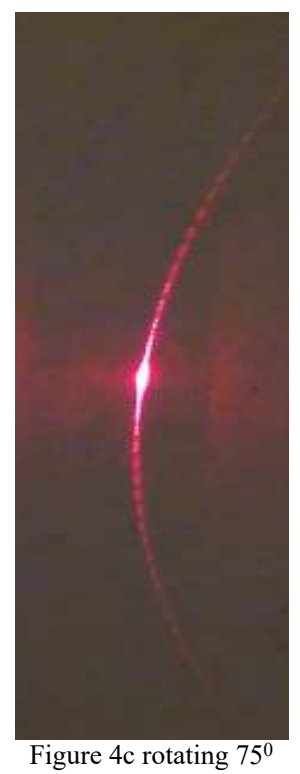

Figure $4 \mathrm{c}$ rotating $75^{0}$

Observation: the interference patterns curved towards the right side that is opposite to that of the curved interference pattern created by rotating the double slit clockwise.

Discussion: in the regular double slit experiment, photons only need to know one factor: whether there is a double slit or not. Now we show that photons also need to know two more factors: (1) the orientation of the double slit and (2) the direction of rotation, i.e., clockwise or counterclockwise. 


\subsubsection{Rotating Diaphragm of Double Slit Around Y-Axis Continuously (Appendix-1 Video)}

Let us place the double slit at the position that the double slit rotates $75^{\circ}$ from the original orientation counterclockwise. Turning on the laser source, we observed the curved interference patten. Then rotating the double slit clockwise continuously and the curved pattern continuously change, the curvature of the interference pattern becomes larger and larger. We reach a position that the laser light is perpendicular to the plane of the double slit, which we referred as the original position, and at that position, the curved interference pattern becomes the regular straight interference pattern. Then continuously rotating the double slit clockwise. The interference pattern starts to curve again but to the opposite direction. See the attached Appendix-1 Video.

\subsubsection{Rotating Diaphragm of Cross-Double Slit Around Y-axis}

\section{(A) Three Double Slits Crossing}

Experiment-3 (Figure 5, 6, 7, 8): the left of Figure 5 shows the diaphragm rotating $0^{0}$ around Y-axis, referred as the original orientation. Its interference pattern is the right of Figure 5.
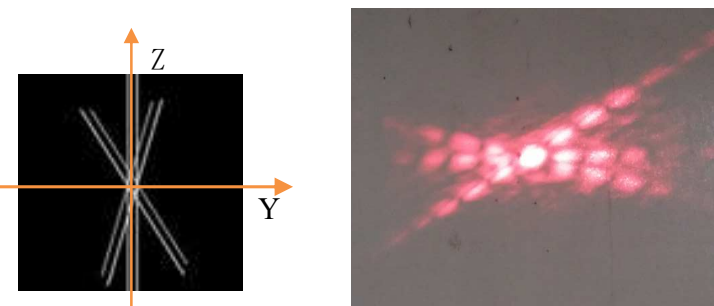

Figure 5 Original Orientation: Rotating $0^{0}$

Let us rotate the diaphragm $45^{\circ}, 60^{\circ}$ and $75^{\circ}$ respectively around $\mathrm{Y}$-axis.

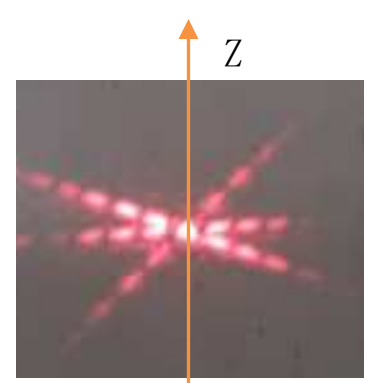

Figure 6 Rotating $45^{0}$

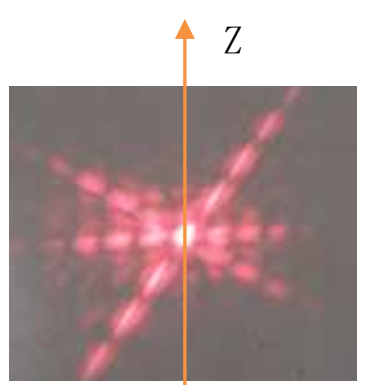

Figure 7 Rotating $60^{\circ}$

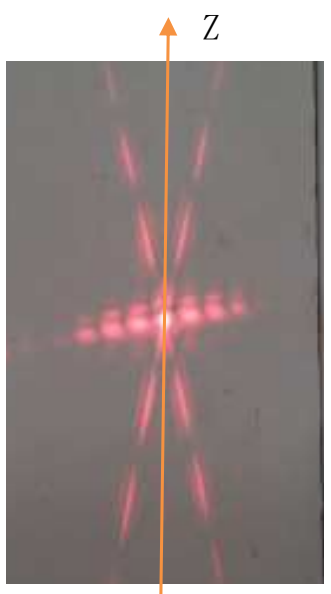

Figure 8 Rotating $75^{0}$

Observation: (1) Figure 6, Figure 7 and Figure 8 show the interference patterns created by the double slit rotating $45^{\circ}, 60^{\circ}$ and $75^{\circ}$ around $\mathrm{Y}$-axis respectively; (2) The angles between two interference 
patterns created by a tilt double slit and vertical double slit become larger and larger when the rotating angle of the diaphragm becomes larger and larger; (3) The interference patterns created by the tilt double slits tend to turn closer to Z-axis.

Photons' behavior depend on which slit they passing through.

Discussion: We show that the interference patterns of the double slit and cross-double slit depend on the orientation of the diaphragms. It is a challenge to interpret the experiments consistently.

\section{(B) Two Double Slits Crossing}

Experiment-4 (Figure 9, 10, 11): the left of Figure 9 shows the diaphragm rotating $0^{0}$ around Y-axis, referred as the original orientation. Its interference pattern is the right of Figure 9.
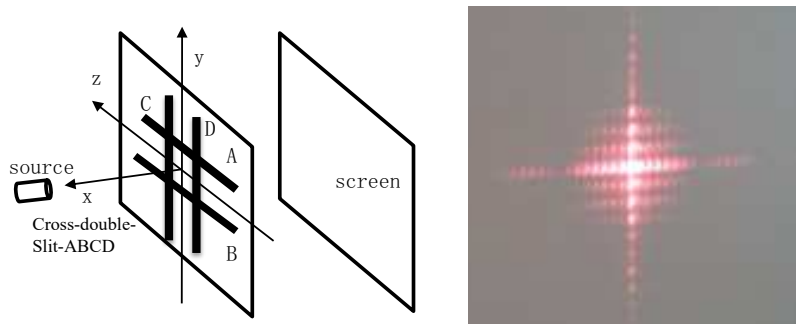

Figure 9 Schematic drawing of cross-double slit and its regular interference pattern

Note that the spacing between slit-A and slit-B is different with that between slit-C and slit-D. The schematic drawing in Figure 9 is not to scale.

Rotating the cross-double slit-ABCD clockwise around Y-axis with different discrete angles.

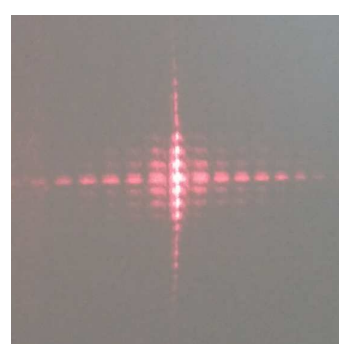

Figure 10 rotating $75^{0}$ clockwise

Observation (Figure 10): at $75^{\circ}$, we observe: (1) the fringes created by the double slit-CD is separated wider; (2) the interference pattern is curved towards left side.

Rotating the cross-double slit-ABCD counterclockwise around $\mathrm{Y}$-axis with different angles.

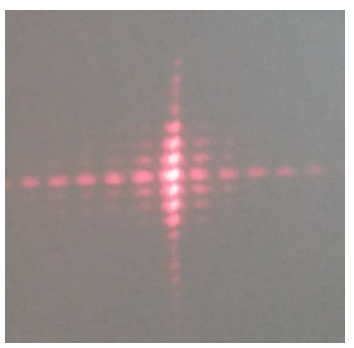

Figure 11 rotating $75^{0}$ counterclockwise 
Observation (Figure 11): at $75^{\circ}$, we observe: (1) the fringes created by the double slit-CD is separated wider; (2) the interference pattern is curved towards right side.

Discussion: The regular double slit and cross-double slit experiments create the straight interference patterns, which are the mystery. In this article, we show that the interference patterns of the double slit and cross-double slit depend on the orientation of the diaphragms. More specifically, the interference patterns are bended by rotating the diaphragms around $\mathrm{Y}$-axis.

It is a challenge to interpret the phenomena/experiments consistently.

\subsubsection{Rotating Diaphragm of Triple Slit Around Y-axis}

The rotations of the diaphragm of the triple slit create the curved interference patterns.

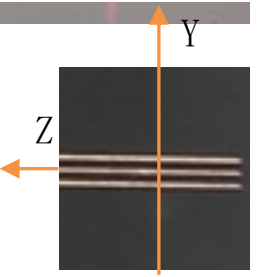

(a)

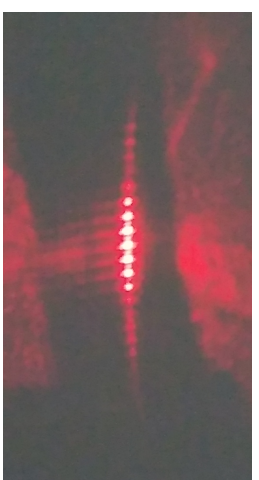

(b)

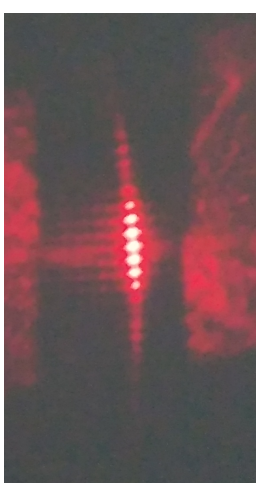

(c)

Figure 12 Interference patterns of rotating triple slit

Figure $12 \mathrm{~b}$ shows the pattern curved towards right attributed to the counterclockwise rotating of the diaphragm. Figure $12 \mathrm{c}$ shows the pattern curved towards left attributed to the clockwise rotating of the diaphragm.

\subsubsection{Rotating Diaphragm of Single Slit Around Y-axis}

Rotating $75^{\circ}$ of the single slit, there is no noticeable curve of the pattern (Figure 13).

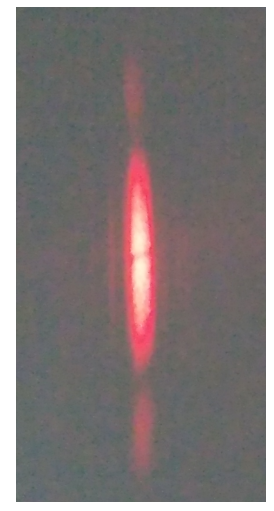

Figure 13 Patter of single slit rotating $75^{0}$ 
Let us derive the Orientation-dependence Formular first. Then we use the formular to describe the experiment and show that the experiments support the derived formular.

\subsubsection{Derivation of Orientation-dependence Formular of Interference Pattern of Double Slit}

The schematic drawing is the following.

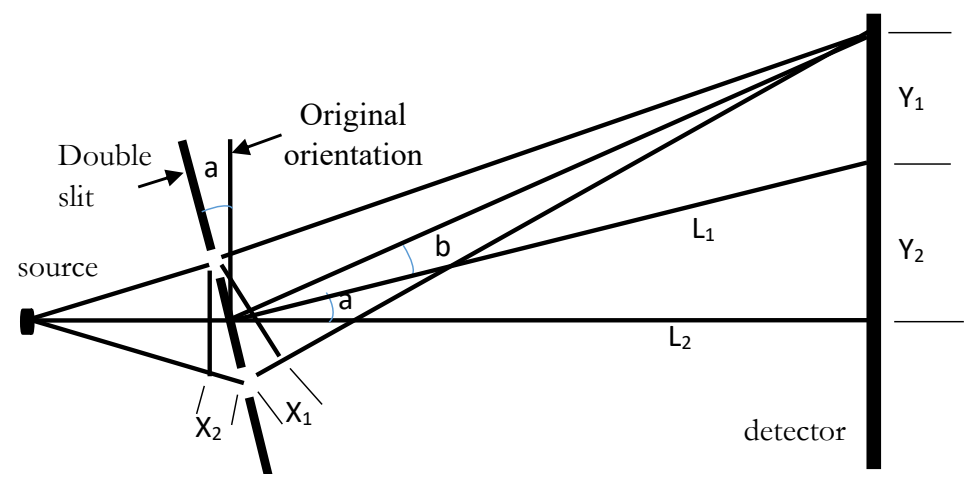

Figure 14 Schematic drawing for derivation of orientation-dependence of double slit experiments

The path difference between two waves passing through two slits respectively is $\left(x_{1}+x_{2}\right)$. The requirement of the interference of two waves is that the path difference satisfies the following relation,

$$
x_{1}+x_{2}=m \lambda
$$

where,

$$
\begin{aligned}
& x_{1} \approx d \sin b, \\
& x_{2} \approx d \sin a, \\
& \sin a=\frac{y_{2}}{L_{1}}=\frac{y_{2}}{\sqrt{L_{2}^{2}+y_{2}^{2}}}=\frac{y_{2}}{L_{2}} \frac{1}{\sqrt{1+y_{2}^{2} / L_{2}^{2}}}, \\
& \sin b \approx \tan b \approx \frac{y_{1}}{L_{1}} .
\end{aligned}
$$

Substituting Eq. (2), Eq. (3), Eq. (4) and Eq. (5) into Eq. (1), we obtain the equation of the orientation-dependence of the constructive/destructive interference pattern of the double slit,

$$
\begin{aligned}
& y \equiv y_{1}+y_{2}=\frac{m \lambda}{d} L_{2} \sqrt{1+(\tan a)^{2}}, \\
& y=\left(m+\frac{1}{2}\right) \frac{\lambda}{d} L_{2} \sqrt{1+(\tan a)^{2}},
\end{aligned}
$$

where, $(\tan a)^{2}=\frac{y_{2}^{2}}{L_{2}^{2}}$, "y" is the position of a bright/dark fringe from the zeroth-order fringe.

\subsubsection{Rotating Diaphragm of Double Slit Around Z-axis}

The experiments show the orientation-dependence of the interference patterns visually.

\section{Experiment-5:}

(A) The diaphragm at the original orientation and its interference pattern (Figure 15). 


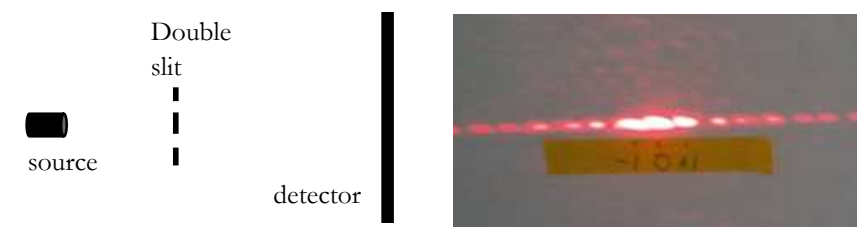

Figure 15 The diaphragm at original orientation and its pattern Equation (6) gives $y=\frac{m \lambda}{d} L_{2}$, which is the normal situation.

(B) The diaphragm rotates $30^{\circ}$ from the original orientation (Figure 16).

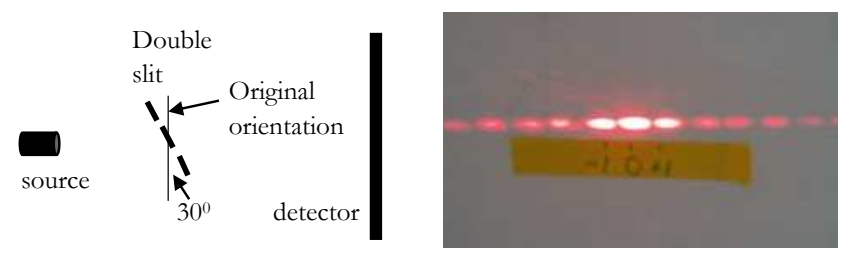

Figure 16 The diaphragm rotates $30^{\circ}$ and its pattern

Eq. (6) gives $y \approx 1.15 \frac{m \lambda}{d} L_{2}$.

(C) The diaphragm rotates $45^{0}$ from the original orientation (Figure 17).

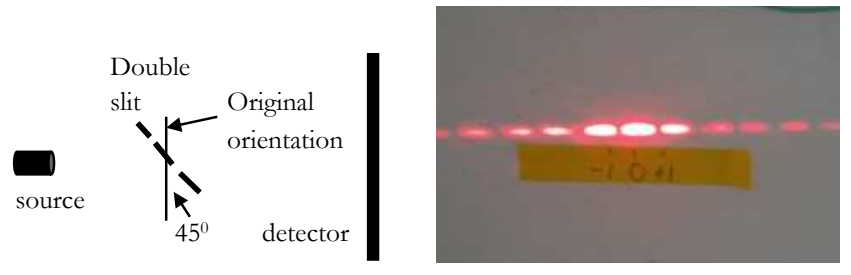

Figure 17 The diaphragm rotates $45^{0}$

Eq. (6) gives $y \approx 1.4 \frac{m \lambda}{d} L_{2}$.

(D) The diaphragm rotates $60^{\circ}$ from the original orientation (Figure 18).

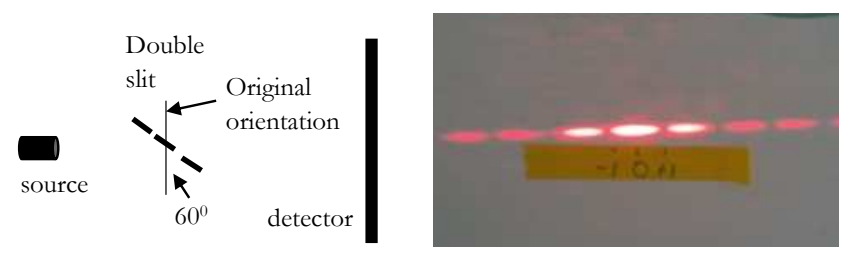

Figure 18 The diaphragm rotates $60^{\circ}$

Eq. (6) gives $y \approx 2 \frac{m \lambda}{d} L_{2}$.

(E) The diaphragm rotates $75^{0}$ from the original orientation (Figure 19).

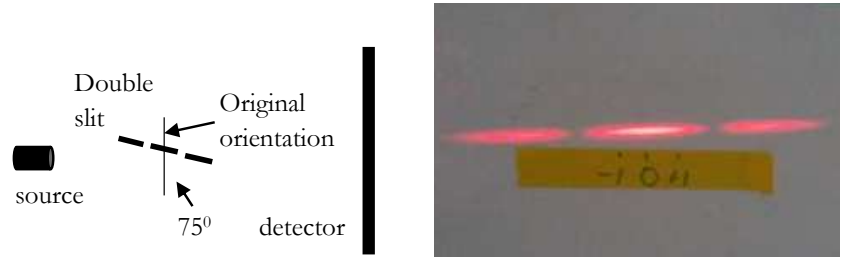

Figure 19 The diaphragm rotates 75 degrees

Eq. (6) gives $y \approx 3.86 \frac{m \lambda}{d} L_{2}$. 
The experimental results support the derived formular (6) describing the orientation-dependence of the interference patterns. Figure 13 to figure 19 show the evolution of the interference pattern varying with angles of the double slit rotating around the z-axis.

\subsubsection{Rotating Diaphragm of Cross-Double Slits Around Z-axis}

The cross-double-slit apparatus consists of source, cross-double-slit, protractor and screen.

\section{(A) Two Double Slit crossing}

Let us consider a simple cross-double slit that consists of double-slit-AB and double-slit-CD, and double-slit-AB is perpendicular to double-slit-CD (Figure 20).
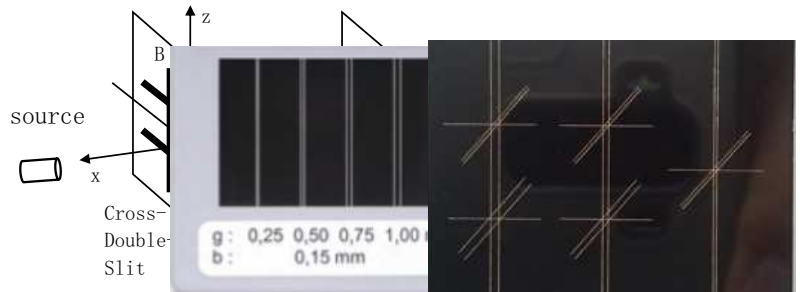

Figure 20 Cross-double-slit apparatus

We have shown that each double slit of the cross-double slit creates the interference pattern independently [7] [8] [9]. The pattern of the cross-double slit-ABCD is the combination of the two patterns created by the double slit-AB and double slit-CD.

The patterns created by rotating around $\mathrm{Y}$-axis and around $\mathrm{Z}$-axis are the same, since the double slit-AB is identical with the double slit-CD, but rotating 90 degrees. So we only consider the situation of rotating around Z-axis.
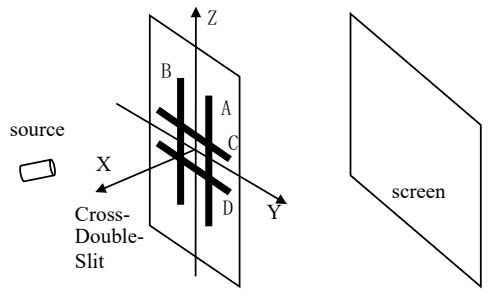

Figure 21 Cross-double-slit rotating around z-axis

Experiment-6 (Figure 22-26): The cross-double slit at the original orientation.

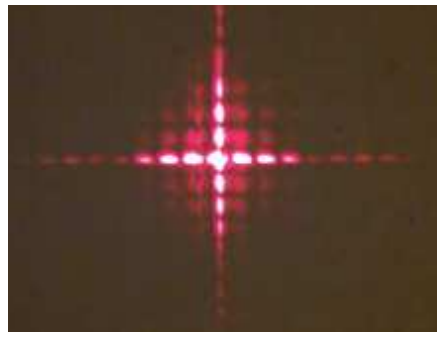

Figure 22 Diaphragm at $0^{0}$

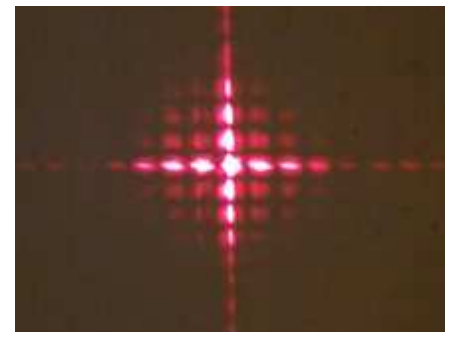

Figure 23 Diaphragm rotates $30^{\circ}$ 
The diaphragm rotates $30^{\circ}$ around Z-axis (Figure 23).

The diaphragm rotates $45^{\circ}$ around Z-axis (Figure 24).

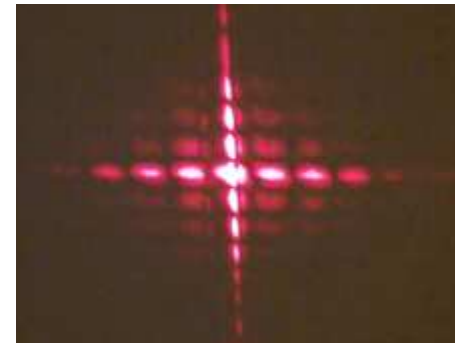

Figure 24 Diaphragm rotates $45^{0}$

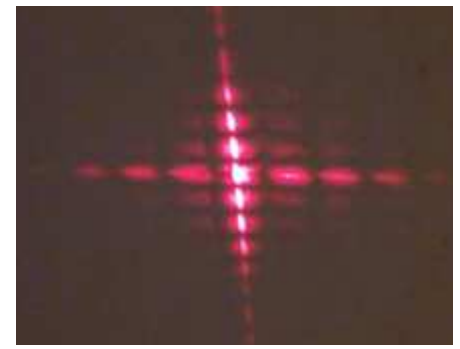

Figure 25 Diaphragm rotates $60^{\circ}$

The diaphragm rotates $60^{\circ}$ around Z-axis (Figure 25).

The diaphragm rotates $75^{\circ}$ around Z-axis (Figure 26).

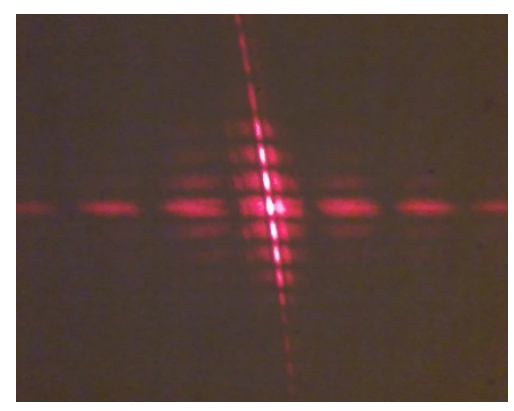

Figure 26 Diaphragm rotates $75^{0}$

Conclusion: The interference patterns of double slit-AB have the same orientation-dependence as shown in Figure 23 to Figure 26. While the interference patterns created by double slit-CD at different rotation-angles curved differently.

\section{(B) Six Double Slits Crossing}

Now consider a cross-double slit that consists of six double slits crossing to each other (Figure 27). The Z-axis is between two vertical double slits and parallel to them. The laser source is on the normal vector of the cross-double slit at the original orientation. Here we consider the rotation of the cross-double slit around the Z-axis.
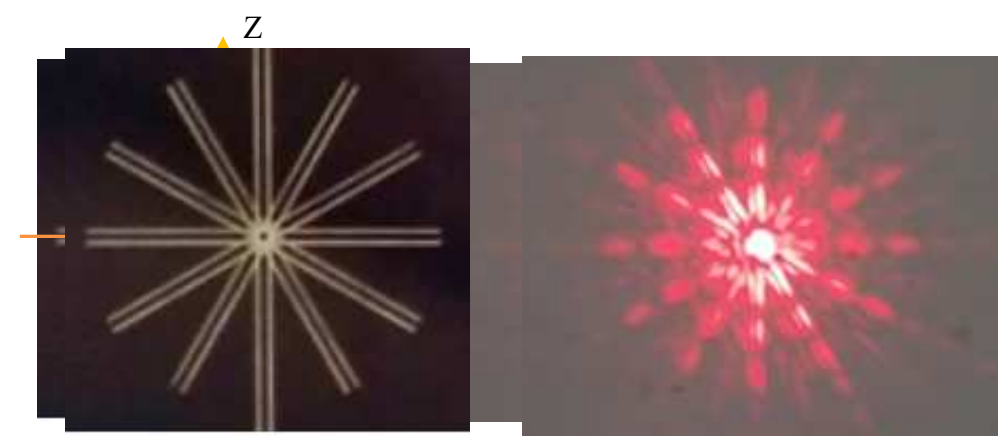

Figure 27 Cross-double slit and its pattern: Rotating $0^{0}$

Experiment-7 (Figure 28, 29): 
The cross-double slit rotates $60^{\circ}$ around Z-axis (Figure 28).

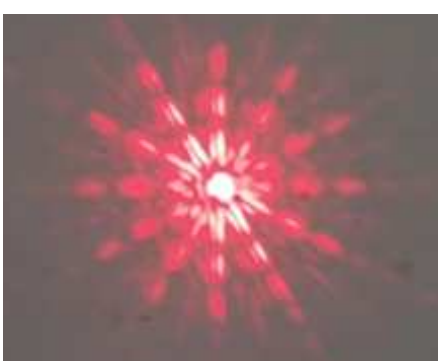

Figure 27 Rotating $0^{0}$

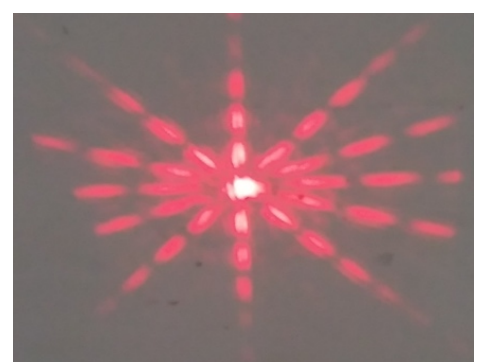

Figure 28 Rotating $60^{\circ}$

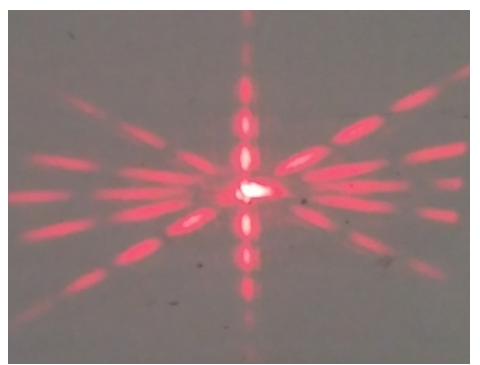

Figure 29 Rotating $75^{0}$

The cross-double slit rotates $75^{0}$ around the Z-axis (Figure 29).

For comparison, we put Figure 27, Figure 28 and Figure 29 side by side, which clearly show the difference/evolution.

Observation: when the rotating angle becomes larger: (1) the distances between two fringes of patterns created by non-horizontal double slits become larger; (2) the patterns created by the tilt-double slits tilt towards the horizontal interference pattern; (3) the distance between fringes created by the vertical double slit is largest; (4) Figure 29 shows the vertical patterns created by the horizontal double slit slightly curved.

Discussion: The interference patterns created by the tilt double slits tend to get closer to Y-axis

\section{(C)Three Double Slits Crossing}

Now to show the orientation-dependence of the interference pattern of the tilt-double slit, especially the novel phenomena that the patterns created by the tilt-double slits tilt towards the horizontal pattern, let us consider two double slits crossing at $15^{\circ}$ and $30^{\circ}$ to the vertical slit respectively (Figure 30). The laser source is on the normal vector of the diaphragm for the original orientation, rotating $0^{0}$. When the diaphragm rotates, the laser source stay.

Experiment-8 (Figure 30, 31 and 32): three double slits cross to each other at $15^{\circ}$ and $30^{\circ}$. The diaphragm is at the zero-degree position.
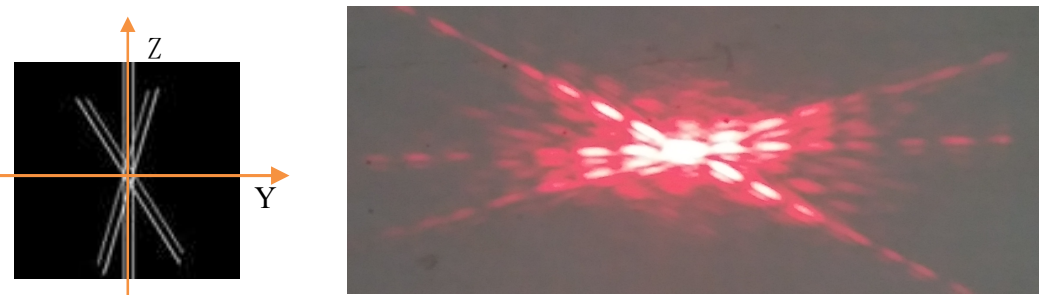

Figure 30 Rotating $0^{0}$

Observation: the interference patterns perpendicular to the double slits created them, respectively. The angles between the slits are the same as that between the interference pattens.

Experimental Step-1 (Figure 31): the diaphragm rotates $60^{\circ}$ around Z-axis. 


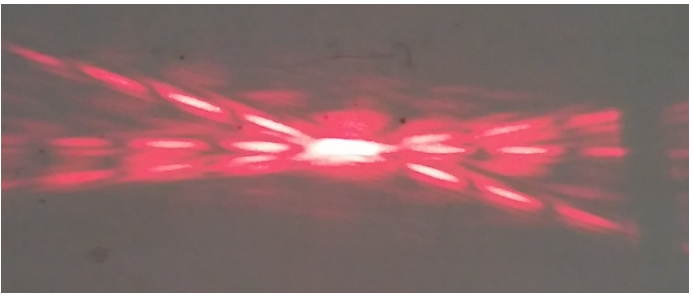

Figure 31 Rotating $60^{\circ}$

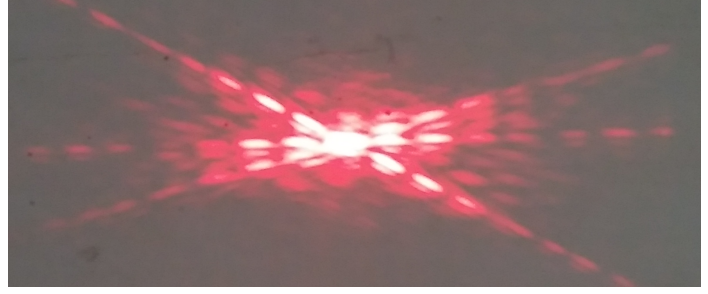

Figure 30 Rotating $0^{0}$

Observation (Figure 31): the angles between two interference patterns are smaller than that shown in

Figure 30. The distances between two fringes of the same patten are larger than that in Figure 30.

Experimental step-2 (Figure 32): the diaphragm rotates $75^{\circ}$ around Z-axis

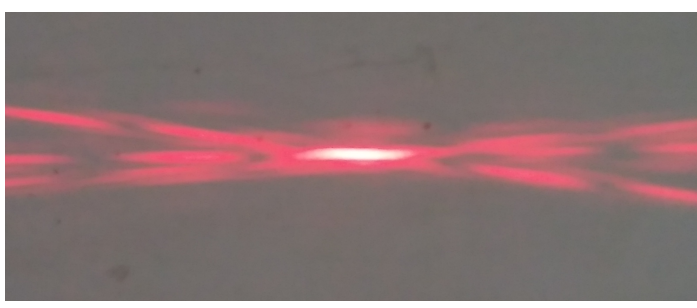

Figure 32 Rotating $75^{0}$

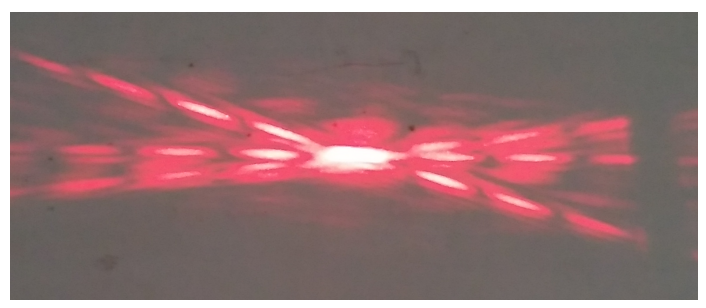

Figure 31 Rotating $60^{0}$

Observation (Figure 32): the angles between two interference patterns are smaller than that shown in Figure 31. The distances between two fringes are larger than that shown in Figure 31.

Conclusion: The interference patterns created by two tilt double slits tend to get closer to Y-axis.

\subsubsection{Rotating Diaphragm of Triple Slit Around Z-axis}
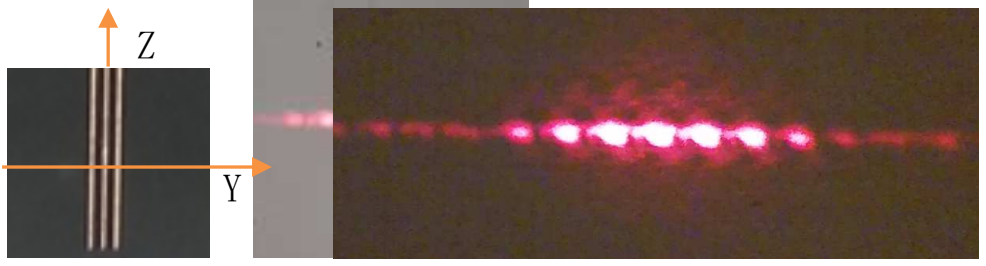

Figure 33 Triple slit and pattern: rotating $0^{0}$

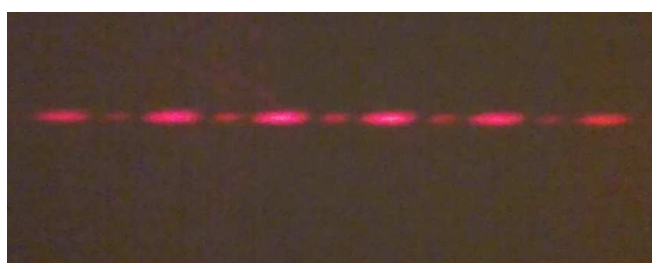

Figure 34 triple slit and pattern: rotate $75^{\circ}$

The triple slit rotating around Z-axis causes the same phenomena that the distances between fringes are extended.

\subsection{Rotating Diaphragm Around X-Axis: Rotation-Invariance}

\subsubsection{Rotating Diaphragm of Double Slit Around X-axis}

We consider two situations: discrete and continuous rotations. 
First situation: discrete rotation.

Experiment-9: (Figure 35). Rotating the double-slit clockwise a discrete angle, for example, 15 degrees.
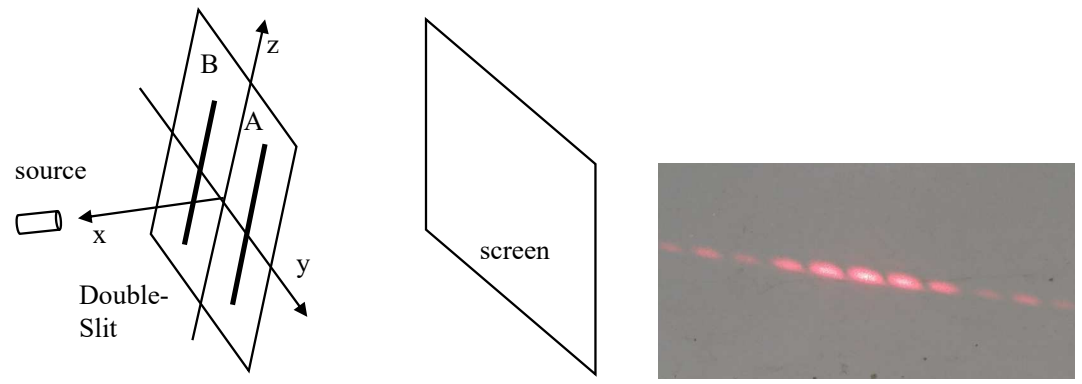

Figure 35 Double-slit rotates around x-axis at angle and its pattern

Observation: the pattern rotates the same angle clockwise and without change.

The interference pattern is perpendicular to the double slit.

Second situation: continuous rotation

The combination of the interference patterns of the cross-double slit experiments with different number of double slits crossing to each other suggests that the double slit and cross-double slit have rotation-invariance around their normal vectors. If we increase the number of double slits that intersect at the same spot, the shape of the intersection will approach a circular disc, each slit is tangent to the intersection and forms disc-3, which is surrounded by ring-2 (Figure 36a). The zero-order fringe rotates at the same spot, and forms a bright fringe 0 , while the rotation of its first-, second- and third-order fringes form bright rings 1, the dark fringes form dark rings 2, and so on (Figure 36b).

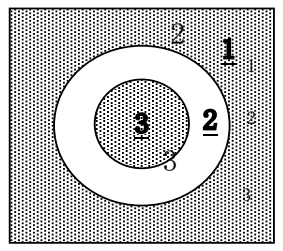

(a)

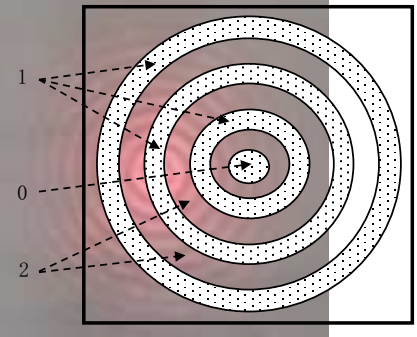

(b)

Figure 36 Predicted pattern of continuously rotating double slit/cross-double slit around $\mathrm{x}$-axis

The above prediction has been tested by the experiment.

Experiment-10 (Figure 37): The apparatus consisting of obstacle 1 with a hole, disc 3 placed at the center of hole-2 and leaving ring-shape gap 2 between disc 3 and obstacle 1 (Figure 37). 

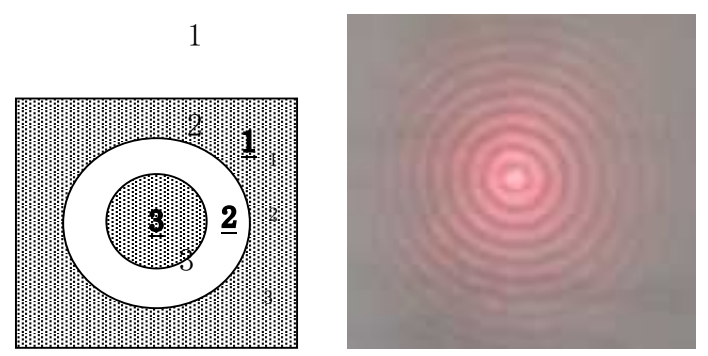

Figure 37 Rotation symmetry of double slit/cross-double slit

Observation: Figure 37 indicates that double slit and cross-double slit have rotation-invariance around their normal vector.

\subsubsection{Rotating Diaphragm of Cross-Double Slit Around X-axis}

Experiment-11 (Figure 38 and Figure 39):

Experimental Setup-1 (Figure 38): Using the cross-double slit that consisting of three double slit crossing to each other at angle $15^{\circ}$ and $30^{\circ}$ respectively. We define $\mathrm{X}$-axis being perpendicular to the plane of the diaphragm and point out the plane.
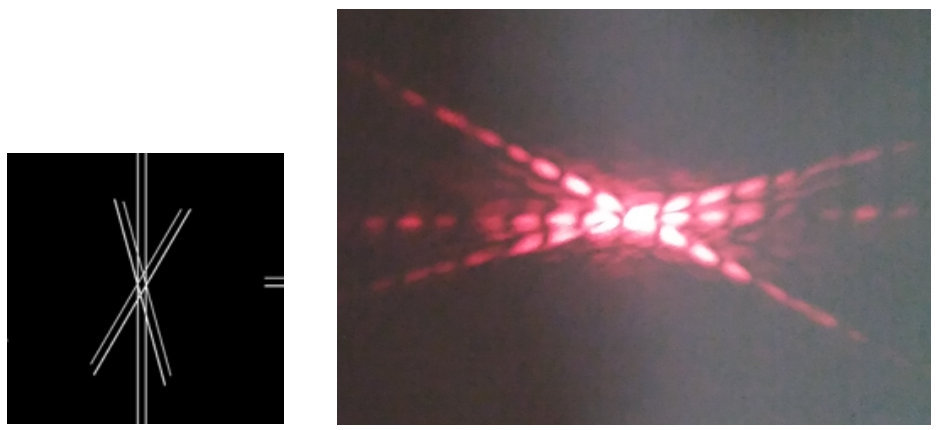

Figure 38 Cross-double slit and its interference pattern

Observation: three interference patterns cross at the same spot with the angles that are the same as that between three double slits respectively.

Experimental Setup-2 (Figure 39): Then rotating the diaphragm $90^{\circ}$ around X-axis.
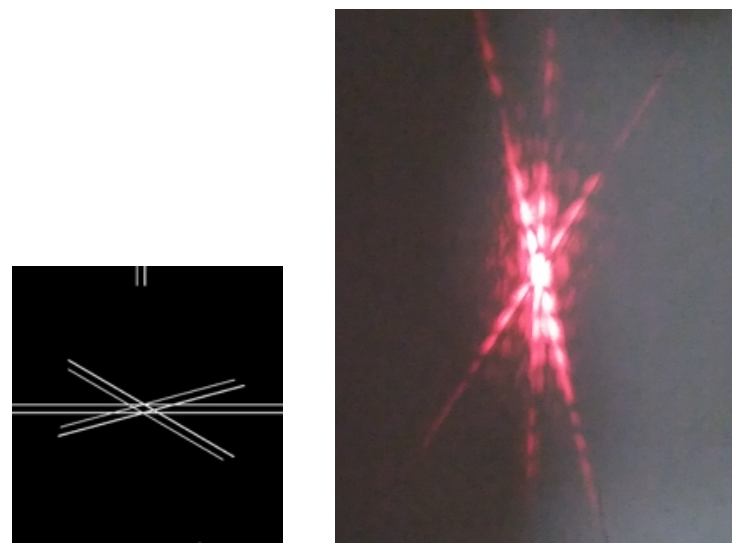

Figure 39 diaphragm rotating $90^{\circ}$ around $\mathrm{X}$-axis counterclockwise and its pattern 
Observation: the interference pattern rotating $90^{\circ}$ counterclockwise.

Conclusion: the cross-double slit has rotation-invariance around their normal vector, $\mathrm{X}$-axis.

\section{Summary}

We show the novel mystery phenomena of the double slit/cross-double slit/triple slit experiments:

(1) the characteristics of the interference patterns of the double slit/cross-double slit/triple slit experiments depend on the orientation of the diaphragm used in the experiment, i.e., the rotation around which axis, the rotation angle, the rotation direction (clockwise or counterclockwise); and (2) in the cross-double slit experiments, the characteristics of the interference patterns also depend on which slit photons passed through; (3) unlike the regular double slit/cross-double slit/triple slit experiments, in which the light is perpendicular to the diaphragm and the interference patterns are along the straight line, the interference patterns of the double slit/cross-double slit/triple slit experiments can be along curve, and the curvatures depend on the orientations of the diaphragms of the double slit/cross-double slit/triple slit.

Now we have comprehensive phenomena/data to be interpreted and described. Those experiments provide comprehensive data for developing/testing a theoretical model.

In the regular double slit experiment, photons only need to know one factor that is whether there is a double slit. Now photons also need to know more factors: the orientation of the double slit. In the cross-double slit experiments, photons also need to know which slit they passed through.

\section{Appendixes}

\section{A-1 Video: Evolution of Curved Interference Pattern of Double Slit Experiment}

\section{A-2 Novel Multi-Slits for Exploring Mystery of Cross-Double Slit}

To explore the mystery of the double slit/cross-double slit, we designed novel multi-slits in which a cross-double slit is divided into different parts/sections to test the function of each part/section of the cross-double slit. The widths of every slit are the same, and spacing between all of vertical double slits and between all of horizontal double slits are the same.

The normal Cross-double-slit- $\mathrm{ABCD}$, the double slit-AB perpendicular to the double slit- $\mathrm{CD}$, is shown in Figure A1 for the purpose of comparation.

The novel multi-slits are shown below.

Novel Multi-Slits-1: Figure A2 shows the intersection of the cross-double slit-ABCD. The purpose: testing whether the intersection of the cross double slit creates the whole interference pattern. 


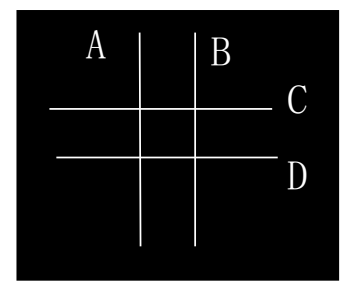

Figure A1

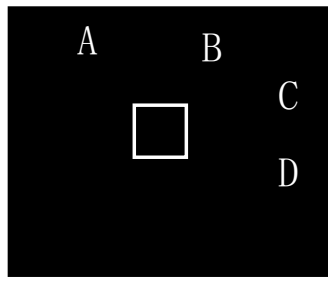

Figure A2

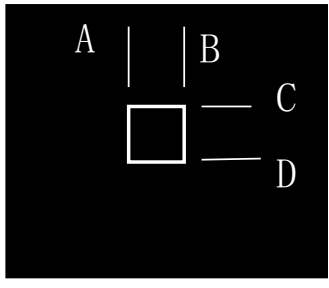

Figure A3

Novel Multi-Slits-2 (Figure A3): there are gaps between the intersection and the parts of slit-A, -B, -C and -D.

Novel Multi-Slits-3 (Figure A4)

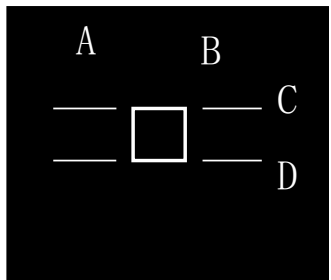

Figure A4

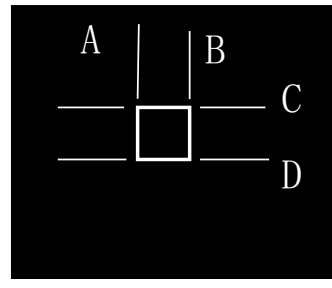

Figure A5

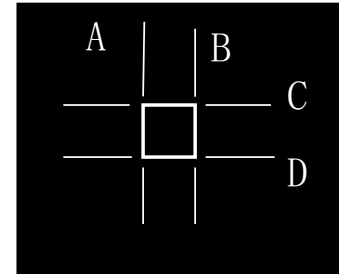

Figure A6

Novel Multi-Slits-4 (Figure A5).

Novel Multi-Slits-5 (Figure A6).

Novel Multi-Slits-6 (Figure A7).

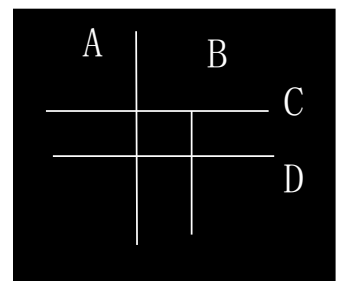

Figure A7

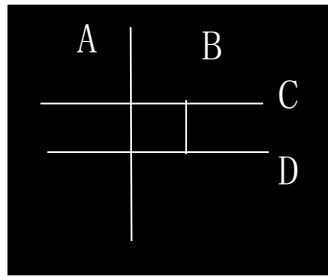

Figure A8

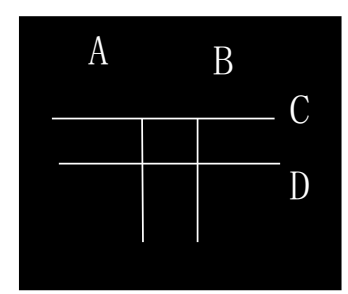

Figure A9

Novel Multi-Slits-7 (Figure A8)

Novel Multi-Slits-8 (Figure A9)

Novel Multi-Slits-9 (Figure A10)

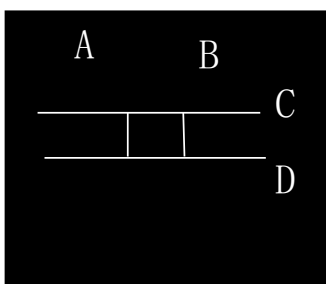

Figure A10

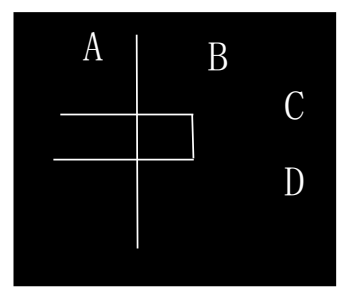

Figure A11

Novel Multi-Slits-10 (Figure A11)

Novel Multi-Slits-11 (Figure A12) 


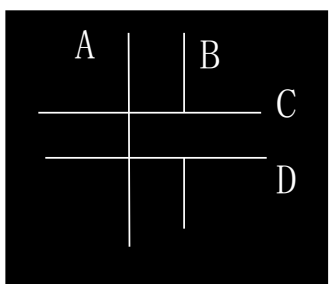

Figure A12

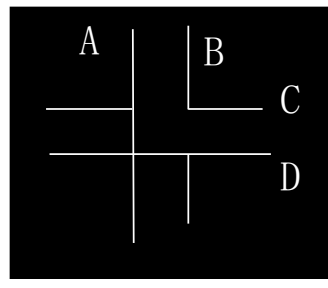

Figure A13

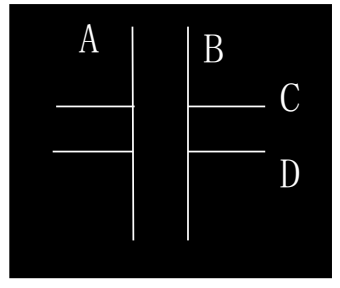

Figure A14

Novel Multi-Slits-12 (Figure A13)

Novel Multi-Slits-13 (Figure A14)

Novel Multi-Slits-14 (Figure A15)

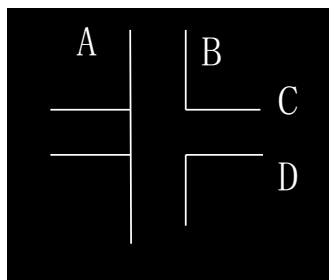

Figure A15

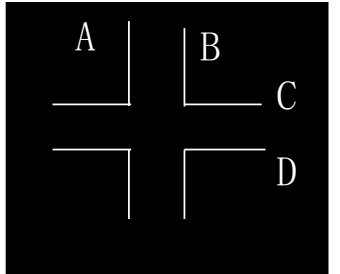

Figure A16

Novel Multi-Slits-15 (Figure A16)

\section{References}

[1] A. Ananthaswamy, “Through Two Doors at Once”, Dutton, New York, NY, (2018).

[2] A. Robinson, “The Last Man Who Knew Everything”. New York, NY: Pi Press., (2006).

[3] R. Feynman, R. Leighton, and M. Sands, “The Feynman Lectures on Physics” (Addison-Wesley, Reading, 1966), Vol. 3.

[4] S. Rashkovskiy, Is a rational explanation of wave-particle duality possible? arXiv 1302.6159 [quant-ph] 2013.

[5] Hui Peng, "Novel Double Slit and Cross-Double Slit Experiments: Interference Patterns Depending on Orientations of Diaphragms", Research Square, preprint, DOI: 10.21203/rs.3.rs-653201/v1, 2021.

[6] Hui Peng, "Curved Interference Pattern---Novel Double Slit and Cross-Double Slit experiment (2)", Research Square, preprint, DOI: https://doi.org/10.21203/rs.3.rs-677223/v1, 2021.

[7] Hui Peng, “Cross-Double-Slit Experiment and Extended-Mach-Zehnder Interferometer”, open-science-repository.com/physics-45011872.html, 2019.

[8] Hui Peng, “Observations of Cross-Double-Slit Experiments.” International Journal of Physics, vol. 8, no. 2: 39-41. DOI: 10.12691/ijp-8-2-1, 2020.

[9] Hui Peng, "Double Slit to Cross Double Slit to Comprehensive Double Slit Experiments", Research Square, preprint, DOI: https://doi.org/10.21203/rs.3.rs-555223/v1, 2021. 


\section{Supplementary Files}

This is a list of supplementary files associated with this preprint. Click to download.

- 20210630evolutionofcurvedinterferencepattern.mp4 